\title{
Changes in blood insulin resistance, GLUT4 \& AMPK after continuous and interval aerobic training in normal and diabetic rats
}

\author{
Mohammad Esmaiel Afzalpour ${ }^{\mathrm{a}^{*}}$, Mohammad Reza Yousefi ${ }^{\mathrm{b}}$, Seyed Hossein Abtahi Eivari ${ }^{\mathrm{c}}$, Saeed Ilbeigi $^{\mathrm{d}}$ \\ ${ }^{a}$ Full Professor, Department of Physical Education and Sport Sciences, University of Birjand, Birjand, Iran. ${ }^{\mathrm{b}}$ Ph.D Student in Exercise Physiology, \\ University of Birjand, Birjand, Iran. ${ }^{\mathrm{c}}$ Assistant Professor, Department of Clinical Biochemistry, Gonabad University of Medical Sciences, Gonabad, Iran. \\ ${ }^{\mathrm{d}}$ Assistant Professor, Department of Physical Education and Sport Sciences, University of Birjand, Birjand, Iran.
}

\section{ARTICLE INFO}

Article history:

Received on: 04/07/2016

Revised on: 12/07/2016

Accepted on: 18/08/2016

Available online: 03/09/2016

Key words:

Glucose transportertype4, Insulin resistance, Aerobic training, AMP-activated protein kinase.

\begin{abstract}
Objectives: Diabetes mellitus is now seen as a worldwide epidemic disease with prevalent and incidence data. Exercise training is known to promote beneficial changes in diabetic patient.

Materials \& Methods: A number of 60 male rats weighing 180 to 310 grams, 13 weeks old were divided into six groups. The exercise protocol was aerobic training for six weeks. In this study, factors like Insulin resistance, glucose transporter type 4 and AMP-activated protein kinase were measured. The data were analyzed using oneway analysis of variance test in $\mathrm{P}<0.05$ level.

Results: The results showed a significant difference in insulin resistance $\left(\mathrm{P}=0.001, \mathrm{~F}_{5,37}=10.80\right)$, Glucose transporter type $4(\mathrm{P}=0.001, \mathrm{~F} 5,37=20.14)$ and $\mathrm{AMP}$-activated protein kinase $(\mathrm{F} 5,37=16.2, \mathrm{P}=0.001)$ levels among different groups. There was no significant difference between the impact of continuous and interval training on these indices $(\mathrm{P}=1.00)$.

Conclusions: The continuous and interval training by increasing the Glucose transporter type4 protein content and AMP-activated protein kinase lead to reduced blood glucose levels and improved insulin resistance. Furthermore, we could use conducting interval training as a therapeutic approach to manage diabetes for the participants who were not able to perform the continuous training due to fatigue.
\end{abstract}

\section{INTRODUCTION}

The diabetes mellitus is a metabolic disorder that is widespread in the world (Abou-Seif and Youssef 2004). The chronic high blood sugar has many complications which lead to damages in various organs and disorder in their function including 10 years less lifetime (Association, 2004) 10 times more common amputation (Kastorini and Panagiotakos 2010), 9\% death (Kastorini and Panagiotakos 2010), and visual impairment and blindness (Dabelea et al., 1998). There is direct relationship between chronic complications of diabetes and high level of blood glucose (Gomez-Perez et al., 2010). Today, there is no doubt that improved glycemic control in diabetic patient

* Corresponding Author

Postal address: No 6, 5 Mirdamad Street, Birjand, Iran.

Email address: mafzalpour @ birjand.ac.ir; Tel:09155614517. results in decreased incidence of chronic complications of this disease (Association, 2004). The transportation of glucose into the muscle fiber is done by glucose transporter proteins (GLUTs); the Glucose transporter type4 (GLUT4) is the most important glucose transporter isoform in skeletal muscles. The insulin and exercise through different paths stimulate fast and intense GLUT4 translocation to plasma membrane level and cause glucose uptake in muscle and adipose tissue (Augustin, 2010). The binding Insulin to its receptor causing a series of intracellular reactions which lead to the increase of glucose uptake. Beside of insulin, Exercise Cause an increase glucose uptake. in this action it is believed that exercise cause an increase in AMP-activated protein kinase (AMPK) activity, GLUT4 translocation to the cell surface membrane and finally glucose uptake (Adam et al., 2005). Skeletal muscle plays a major role in maintaining homeostasis of blood glucose. It uses glucose as a major source of energy during contractile activity. 
Exercise can increase skeletal muscle sensitivity to insulin, improve insulin resistance and regulate glucose homeostasis in rat models of type 2 diabetes (Adam et al., 2005). The results of researches show that stimulating AMPK increase glucose uptake (Hawley and Lessard 2008). It is believed that the increased muscle glycogen stores after the exercise is due to increased GLUT4 (Chou et al., 2005; Tsai et al., 2006)and increased GLUT4 protein translocation from inside the cell to plasma surface (Tsai et al., 2006). The exercise-stimulation increases in the translocation of GLUT4 to the skeletal muscle cell membrane occur in people who are healthy and in patients with $\mathrm{T}_{2} \mathrm{DM}^{8}$. In this context, reported that the increased GLUT4 in skeletal muscle of healthy and diabetic rats after 6 weeks running (Park et al., 2011). Moreover, showed that resistance exercise led to $40 \%$ and $21 \%$ increase of GLUT4 and insulin receptors content in diabetic patients' muscles (Holten et al., 2004). Also, showed that 7 week running increases the GLUT4 level and improves insulin resistance in obese rats (Christine et al., 2002). In contrast, showed that there was no change in GLUT4 content of soleus muscle in three weeks of running (Rodnick et al., 1990). Moreover, Reported that the GLUT4 level is decreased due to exercise-induced insulin reduction (Vannucci et al., 1998). Finally, showed that exercise improves insulin sensitivity in independent ways from GLUT4 in diabetic rats (Ivy 2004). AMPK is also different in terms of research results. For instance, Cao et al (2012) (Cao et al., 2012). Musi et al (2001) (Musi et al., 2001) and Chen et al (2003) (Chen et al., 2003) reported an increase in AMPK after training, while McGee et al (2003) (McGee et al., 2003) reported no changes in AMPK after training. AMPK appears to be an important regulator of energy metabolism during skeletal muscle exercise (McGee et al., 2003).

Exercise training compensates for the defects in muscle insulin resistance by increasing expression of GLUT4. This increase in GLUT4 protein is essential for the improvement in muscle insulin resistance (Ivy, 2004).

According to studies conducted in this field and different results in previous studies, this study aims to investigate the impact of two aerobic (continuous and interval) training on AMPK, insulin resistance and GLUT4 indices in soleus muscle of diabetic rats and answer this questions: Do aerobic training is beneficial for diabetics? And what type of training is more effective? Most emphasis of this study is to compare two methods of training, in previous research there is not.

\section{MATERIALS AND METHODS}

A total of 60 male rats weighting between 180 to 310 grams and 13-week old were bought from Kermanshah University of medical science Laboratory Animals Breeding Centre. These animals were kept in a place with 12-hour light-dark cycle, $21 \pm 2$ ${ }^{\circ} \mathrm{C}$ temperature, and a relative humidity of 45 to 55 percent (Kraniou et al., 2004). During the study, the animals were given enough food and water. The subjects were kept for 2 weeks in new conditions to avoid stress and change in physiological conditions. In the second week, the animals were trained to work on the treadmill. The training program was conducted for 5 days (at a speed of 10 meters per minute, once a day for 10 minutes) (Borzykh et al., 2012). After a period of adaptation to laboratory, 30 rats (except healthy control, healthy interval, and healthy continuous groups) got diabetic using Streptozotocin (STZ) drug with a dose of $50 \mathrm{mg}$ per $\mathrm{kg}$ of body weight, solved in citrate buffer 0.1 Molar and acidity 4.5. After a week, the blood samples were taken with a small wound on the tail; the blood glucose levels were measured with a glucometer. After disclosure of diabetes (glucose greater than $300 \mathrm{mg}$ per deciliter) in animals (Srinivasan et al., 2005). The rats were randomly divided into six healthy control, diabetic control, healthy interval, diabetic interval, healthy continuous, diabetic continuous (each group of 10 rats) groups based on the type of training protocol. The training protocol of both continuous and interval groups was six weeks of aerobic training which was performed with progressive intensity and duration and considering gradual overload principle [Table 1]. Throughout the training period, the slope of treadmill was considered zero degree. The training groups ran at the beginning of each training session for 3 minutes at a speed of 7 meters per minute for warming up. The treadmill speed was increased 2 meters per minute to achieve the desired speed. The speed of treadmill was reduced reversely to initial speed at the end of each training session for cooling down.

Table 1: The process of performing training protocols (Shabkhiz et al., 2008).

\begin{tabular}{|c|c|c|}
\hline Week & Interval training & Continuous training \\
\hline 1 & 2repeats for 7 minutes and 12 meters per minute & 14 minutes at a speed of 12 meters per minute \\
\hline 2 & 2 repeats for 9.5 minutes and 12 meters per minute & 19 minutes at a speed of 12 meters per minute \\
\hline 3 & 2 repeats for 12 minutes and 13 meters per minute & 24 minutes at a speed of 13 meters per minute \\
\hline 4 & 2 repeats for 14.5 minutes and 14 meters per minute & 29 minutes at a speed of 14 meters per minute \\
\hline 5 & 3 repeats for 12 minutes and 15 meters per minute & 36 minutes at a speed of 15 meters per minute \\
\hline 6 & 3 repeats for 14.5 minutes and 16 meters per minute & 43.5 minutes at a speed of 16 meters per minute \\
\hline
\end{tabular}


To avoid possible effects of electric shock ( 1 to 3 volts) on study results, the animals were trained through audio stimulation to avoid approaching and resting in end section of device (Siamilis et al., 2009). However, 48 hours after last exercise session followed by 12 hours fasting, the rats were anesthetized with inhaling ether solution in inside of glass chamber. Cutting the skin of abdomen and chest area and opening abdominal cavity, about $10 \mathrm{ml}$ of blood was taken directly from the heart and was transferred to test tube. The collected samples were immediately centrifuged (at $3000 \mathrm{rpm}$ for $10 \mathrm{~min}$ ) and were used to assess the insulin level. After dissection, their soleus muscle was also taken by surgery knife. It was washed, homogenized, and frozen in liquid nitrogen and was kept at $-80^{\circ}$ C for subsequent analyses (Ogonovszky et al., 2005). In the present study, the commercial kit of Sweden Mercodia Company with sensitivity of 0.015 micrograms per Liter were used to measure insulin level. Also the commercial kit of China-American Cosabio Company with sensitivity of 0.04 and 15 ng.per.ml for GLUT4 and AMPK respectively. The samples were analyzed using Sandwich ELISA method. The insulin resistance is opposite to insulin sensitivity. It is not possible to calculate insulin sensitivity by calculating the number of insulin receptors on the cell surface. Therefore, the HOMA-IR formula (Hosseini et al., 2015) was used to calculate insulin resistance changes and insulin sensitivity changes.

HOMA-IR= fasting insulin $($ Micro unit $/ \mathrm{ml}) \times$ fasting glucose $(\mathrm{mg} /$ dl) $\div 405$

The data were analyzed using Statistical Package for Social Sciences (SPSS), version 16. First, the Shapiro-Wilk test was used to determine the type of distribution of data in all groups. According to results of this test, the one-way analysis of variance (ANOVA) was used to determine between-groups changes of dependent variables at $\mathrm{P}<0.05$ level. Also, the Tukey test was used to determine the difference between pairs.

\section{RESULTS}

The results showed a significant difference in insulin resistance levels among different groups $\left(\mathrm{P}=0.001, \mathrm{~F}_{5,37}=10.80\right)$ [Table 2]. Tukey test showed that insulin resistance in diabetic control rat is significantly higher than healthy control group $(\mathrm{P}=0.001)$. However, there was no significant difference between insulin resistance of diabetic continuous and interval training group and healthy continuous and interval training group $(\mathrm{P}=0.99)$, $(\mathrm{p}=0.96)$ (Figure 1).

Also, the continuous $(\mathrm{P}=1.00)$ and interval $(\mathrm{P}=0.96)$ training had not significant effect on insulin resistance level of healthy rats compared to healthy control group; there was no significant difference between the impact of both training on insulin resistance $(\mathrm{P}=1.00)$.

The results showed a significant difference in GLUT4 of soleus muscle in different research groups $\left(\mathrm{P}=0.001, \mathrm{~F}_{5,37}=20.14\right)$. Tukey test showed that GLUT4 in diabetic control is significantly lower than healthy control group $(\mathrm{P}=0.006)$. Although showed that the continuous $(\mathrm{P}=0.001)$ and interval $(\mathrm{P}=0.004)$ training increase GLUT4 in healthy compared to healthy control group, there was no significant difference between the effects of these two type of training $(\mathrm{P}=0.94)$. In addition, GLUT4 in continuous and interval group was significantly higher than diabetic continuous and interval group $(\mathrm{P}=0.001), \quad(\mathrm{P}=0.002)$ (Figure 2). There was no significant difference between GLUT4 in diabetic continuous $(\mathrm{P}=0.81)$, diabetic $(\mathrm{P}=0.002)$ (Figure 2). Training $(\mathrm{P}=0.99)$, and healthy control groups.

The results show that there is a significant difference between AMPK levels of soleus muscle among different groups $\left(\mathrm{F}_{5,37}=16.2, \mathrm{P}=0.001\right)$ (Figure 3$)$. It was determined that AMPK levels in the diabetic rats of control group compared with the healthy control group is lower $(\mathrm{P}=0.008)$. Although show that continuous $(\mathrm{P}=0.016)$ and interval $(\mathrm{P}=0.001)$ compared with the healthy control group, increase the AMPK level among healthy rats. there was no significant difference between two types of training $(\mathrm{P}=0.953)$. In addition, AMPK levels among continuous compared to diabetic continuous group $(\mathrm{P}=0.003)$ and interval compared to diabetic interval group $(\mathrm{P}=0.005)$ are significantly higher.

Also, there is no significant difference among AMPK levels of diabetic continuous $(\mathrm{P}=0.982)$ and diabetic interval $(\mathrm{P}=$ 1.000) compared to healthy control groups.

Table 2: The mean, standard deviation, F and P- values of dependent variables in separate rat groups.

\begin{tabular}{lcccccccc}
\hline Group & $\begin{array}{c}\text { Healthy } \\
\text { control }\end{array}$ & Diabetic control & $\begin{array}{c}\text { Continuous } \\
\text { training }\end{array}$ & $\begin{array}{c}\text { Diabetic continuous } \\
\text { training }\end{array}$ & $\begin{array}{c}\text { Intermitten } \\
\text { training }\end{array}$ & $\begin{array}{c}\text { Diabetic interval } \\
\text { training }\end{array}$ & F & p-value \\
\hline Weight (g) & $290 \pm 26$ & $229 \pm 21$ & $304 \pm 36$ & $270 \pm 25$ & $283 \pm 37$ & $269 \pm 40$ & 4.24 & 0.004 \\
Insulin resistance & $0.169 \pm 0.03$ & $0.334 \pm 0.12$ & $0.169 \pm 0.03$ & $0.159 \pm 0.01$ & $0.172 \pm 0.01$ & $0.150 \pm 0.02$ & 10.80 & 0.001 \\
GLUT4 (Pg/ mg) & $10.75 \pm 2.33$ & $5.82 \pm 1.74$ & $16.49 \pm 1.77$ & $9.24 \pm 2.64$ & $15.42 \pm 2.52$ & $10.08 \pm 3$ & 20.14 & 0.001 \\
AMPK (Pg / mg) & $5.33 \pm 1.27$ & $2.93 \pm 0.83$ & $7.39 \pm 1.15$ & $4.90 \pm 1.49$ & $791 \pm 1.20$ & $5.42 \pm 0.94$ & 16.2 & 0.001 \\
\hline
\end{tabular}




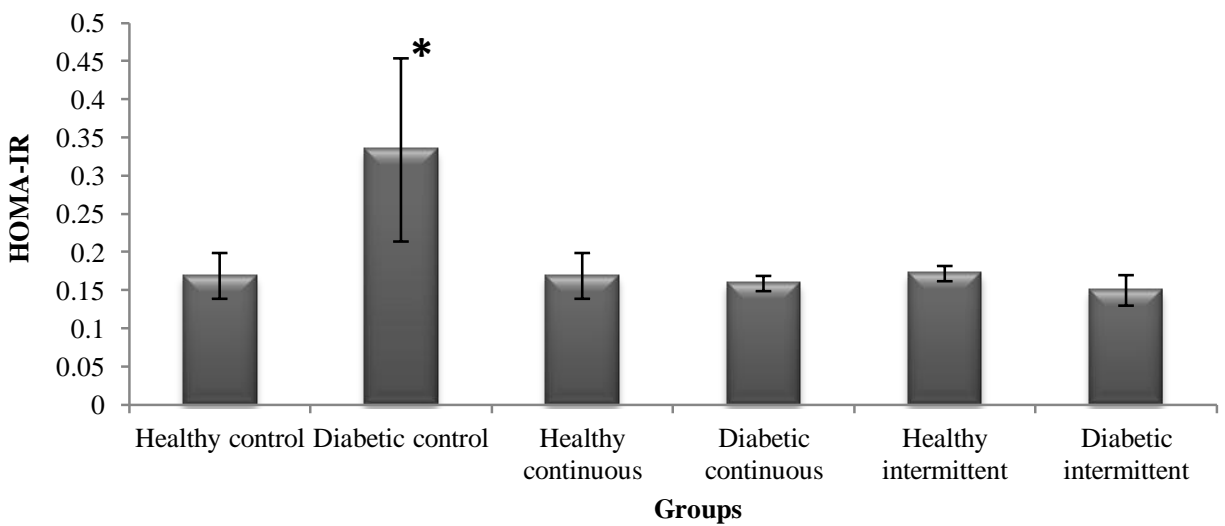

Fig. 1: The figure shows that the insulin resistance level in diabetic control group is significantly $(\mathrm{P}<0.05)$ higher than healthy control group. $*$ Significant difference with control group at $\mathrm{P}<0.05$

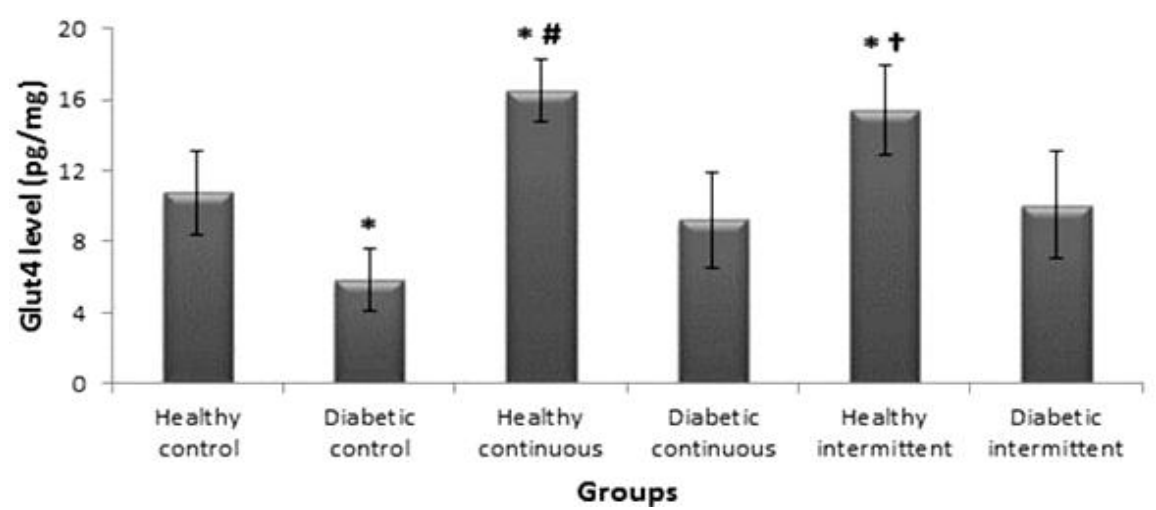

Fig. 2: The comparison of GLUT4 of soleus muscle in healthy and diabetic rats after 6 weeks continuous and interval training. *Significant difference with control group at $\mathrm{P}<0.05$. \# Significant difference with diabetic continuous training group at $\mathrm{P}<0.05 \dagger$ Significant difference with diabetic intermittent training group at $\mathrm{P}<0.05$

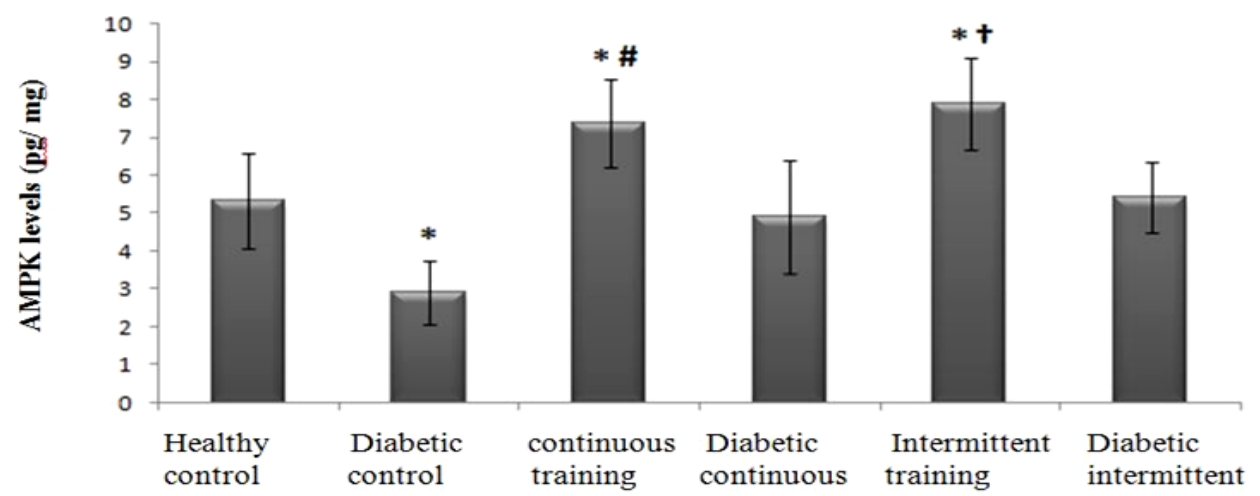

Fig. 3: The comparison of AMPK levels of healthy and diabetic rats after 6 weeks of training. * Significant difference with healthy control group at $\mathrm{P}<0.05$. \# Significant difference with diabetic continuous group at $\mathrm{P}<.05$. $\dagger$ Significant difference with diabetic interval group at $\mathrm{P}<0.05$

\section{DISCUSSION}

The results showed that despite the significant difference between the effect of continuous and interval training on glucose uptake indices and subsequent decrease in blood glucose and increase in Glucose transporter type 4 and AMP-activated protein kinase levels among diabetic rat in different groups compared to normal rat in the corresponding groups, there was no significant difference between the impact of continuous and interval training on these indices. The insulin resistance of diabetic control was higher than healthy control group. However, there was no significant difference between insulin resistance of diabetic continuous and continuous, diabetic interval and interval groups. In addition, the results showed that GLUT4 of soleus muscle in diabetic control was lower than healthy control group. Although the continuous and interval increased GLUT4 in healthy compared to healthy control group. there was no significant difference between these two types of training. In a study conducted on rats, the increased level of GLUT4 in plantaris muscle and no change in soleus muscle was observed after three weeks of training 
(Rodnick et al., 1990). In this context, suggested that due to reduced level of insulin after exercise, GLUT4 was significantly reduced in rabbits (Vannucci et al., 1998). Exercise improves insulin sensitivity, but causes no change in insulin signaling and GLUT4 transmission to membrane surface in muscles of rats. It can be found that the results of present research on GLUT4 are not consistent with above findings. This may be due to difference in training program type and studied muscle. However, the research shows that muscle fiber type is involved in type of response. For example, white fibers are less affected by aerobic activity in terms of GLUT4 and insulin sensitivity. However, the results of present study are consistent with the findings of Durant (2002) increase of GLUT4 transportation to cell membrane (Paula et al., 2002), Caponi et al (2013) increase of GLUT4 (Caponi et al., 2013), Park et al., (2011) increase of GLUT4 (Park et al., 2011), Zarekar et al (2014) increase of GLUT4 and insulin sensitivity (Zarekar et al., 2014), and Catherine et al., (2013) increase of GLUT4 and improved insulin sensitivity (Katharine et al., 2013). The GLUT4 protein is a major mediator for glucose uptake from blood circulation which is expressed in skeletal muscle and adipose tissue (Huang and Czech, 2007). The evidence has shown that the total amount of GLUT4 protein and its displacement to muscle fiber membrane determines the amount of muscle glucose uptake in response to insulin. It is now widely accepted that this protein molecule plays a key role in whole-body insulin sensitivity and glucose tolerance (Hou et al., 2003). There are two routes (insulin and exercise) for stimulating glucose uptake by muscle. During the rest, the glucose uptake by muscle depends on insulin and during exercise, the muscle contraction increases blood glucose uptake. The uptake of glucose into muscle is high even after exercise, because the routes which stimulate glucose uptake remain active for hours after exercise (Colberg et al., 2010). Due to increased GLUT4 protein, increased displacement, and increased exposure of these transport proteins at the cell surface, the muscle contraction increases membrane permeability to glucose and improves insulin action in glucose metabolism (Kiraly et al., 2007). The increased insulin sensitivity after exercise occurs simultaneously with the accumulation of muscle glycogen stores (Hawley and Lessard, 2008). It is believed that increased muscle glycogen stores after exercise is due to increased GLUT4 (Chou et al., 2005, Tsai et al., 2006) and increased GLUT4 protein displacement from inside the cell to plasma surface (Tsai et al., 2006). The findings of some studies show that exercise (TeixeiraLemos et al., 2011; Eguchi et al., 2013) may impact on AMPK activity and AMPK impact on protein GLUT4 (Teixeira-Lemos et al., 2011; Richter and Hargreaves, 2013). However, it can be said that continuous and interval exercises increase AMPK activity and stimulate GLUT4 protein in these groups. In terms of increased insulin sensitivity as one of the findings, it can be said that some mechanisms increase insulin action after aerobic (continuous and interval) exercise including increased insulin receptors signaling, increased GLUT), increased activity of glycogen synthase and hexokinase, decreased release and increased deletion of free fatty acids, increased release of glucose from blood into the muscle due to increased muscle capillaries, and changes in composition of muscle to increase glucose uptake (Hosseini et al., 2015). Given the rise in GLUT4 protein levels in this study, the increased GLUT4 level is probably one of the reasons for improvement of insulin action. Both the insulin dependent and independent routes in diabetic patients are impaired in glucose uptake, because diabetes reduces GLUT4 level and increases resistance to insulin. In contrast, the exercise acts as an alternative to compensate low levels of these indicators. In addition, although diabetes reduces GLUT4 displacement to muscle cell membrane, the exercise compensates this transportation and displacement through stimulating insulin independent route. There is evidence in recent years which shows that AMPK due to its good effects on reducing glucose- one can be used as a good therapeutic tool in the treatment of diabetic patients. AMPK by increasing translocation and gene GLUT4 levels helps to improve glucose uptake in muscles. Also, AMPK reduces synthesis and increases oxidation of fatty acids by phosphorylation and inhibition of acetyl coa carboxylase enzyme. Also, it reduces cholesterol synthesis through inhibiting HMG-COA reductase enzyme. As a result of these actions, it is expected that with phosphorylation and activation of AMPK, insulin function and glucose uptake are improved (Parimai and Ranjan, 2007). In relation to factors activating AMPK, results show that this index is activated in effect of prolonged exercise, creating electrical stimulation in skeleton muscles, anemia in heart muscle, and thermal shock (Parimai and Ranjan, 2007). AMPK, through inhibiting glucose produced by the liver and increasing glucose consumed by muscles, can control the increased levels of glucose in diabetic patients (Parimai and Ranjan, 2007). The results of this research showed that aerobic training (continuous and interval) increases AMPK levels in soleus muscle in diabetic rats. So it can be concluded that, since the level of insulin receptors is reduced as a result of diabetes, reduced level of blood glucose in diabetic patients, as shown in this research, is done through activation of non-insulin dependent path, namely GLUT4AMPK path; and this shows that diabetes can be managed by exercise in diabetic patients even in the case of sharp reduction of insulin and insulin resistance. Unlike previous studies have shown that interval training is most useful for diabetics we concluded that both continuous and interval training were similar effects in improving insulin resistance, GLUT4 and AMPK levels in diabetic rats.

\section{CONCLUSION}

Given that the present study showed that the continuous and interval training impact significantly on GLUT4 protein levels, AMPK and insulin resistance, It seems that training may overcome the damage caused by diabetes on AMPK and GLUT4 protein. However, due to lack of significant difference between the effects of interval and continuous training, the diabetics may perform any of these training to achieve positive results. 
Financial support and sponsorship: Nil.

Conflicts of interest: There are no conflicts of interest.

\section{REFERENCES}

Abou-Seif M, Youssef A. Evaluation of some biochemical changes in diabetic patients Clin ChimActa. 2004;346:161-170.

Adam J, Erik ARa, Richter E. Skeletal Muscle Glucose Uptake During Exercise: How is it Regulated. Physiology. 2005;20:260-270. 2004:11-14.

Association AD. Screening for Type 2 Diabetes. Diabetes Care.

Augustin R. The protein family of glucose transport facilitators: It's not only about glucose after all. IUBMB Life 2010;62: 315-333.

Borzykh A, Kuzmin I, Martianov A, Borovik A, Sharova A, Tarasova $\mathrm{O}$, et al., Changes of rat respiratory and locomotory muscles during aerobic exercise training in continuous and interval regimens. Biophysics. 2012;5:684-689.

Cao S, Li B, Yi X, Chang B, Zhu B. Effects of Exercise on AMPK Signaling and Downstream Components to PI3K in Rat with Type 2 Diabetes. PLoS ONE. 2012;7:51709.

Caponi P, Lehnen A, Pinto G, Borges J, Markoski M, Machado $\mathrm{U}$, et al., Aerobic exercise training induces metabolic benefits in rats with metabolic syndrome independent of dietary changes. Clinics 2013; 68: 1010-1017.

Chen Z, Stephens T, Murthy S, Canny B, Hargreaves M, Witters L. Effect of Exercise Intensity on Skeletal Muscle AMPK Signaling in Humans. Diabetes 2003;52:2205-2212.

Chou C, YLTsai, Hou C, Lee H, Chang W, Lin T. Glycogen overload by postexercise insulin administration abolished the exerciseinduced increase in GLUT4 protein. J Biomed Sci. 2005;12:991-998.

Christine C, Hunt D, Hancock J, Garcia-Macedo R, Mandarino L, Ivy J. Exercise training improves muscle insulin resistance but not insulin receptor signaling in obese zucker rats. J Appl Physiology. 2002;92: 736-744.

Colberg S, Sigal R, Fernhall B, Regensteiner J, Blissmer B, Rubin R. Exercise and type 2 diabetes. Diabetes Care. 2010;33:147-167.

Dabelea D, Hanson RL, Bennett PH, Roumain J, Knowler WC, Pettitt DJ. Increasing prevalence of type 2 diabetes in American Indian children. Diabetologia.1998; 41: 904-910.

Eguchi T, Cumagai K, Fujihara T, Takemasa T, Ozawa T, Numata O. Black tea high-molecular-weight polyphenol stimulates exercise training-induced improvement of endurance capacity in mouse via the link between AMPK and GLUT4. PLoS One. 2013;8:69480.

Gomez-Perez F, Aguilar-Salinas C, Almeda-Valdes P, CuevasRamos D, Garber IL, Rull J. HbA1c for the diagnosis of diabetes mellitus in a developing country. Arch Med Res. 2010;41:302-308.

Hawley J, Lessard S. Exercise training-induced improvements in insulin action. Acta Physiol (Oxf). 2008;192:127-135.

Holten M, Zacho M, Gaster M, Juel C, Wojtaszewski J, Dela F. Strength training increases insulin-mediated glucose uptake, GLUT4 content, and Insulin signaling in skeletal muscle in patients with type 2 diabetes. Diabetes 2004;53:294-305.

Hosseini S, Mir E, Hejazi K, Sayeedi M. The Effect of eight weeks combined training on some insulin resistance markers in middleaged men. Medical Journal of Mashhad University of Medical Sciences. 2015;58:80-86

Hou C, Chou S, Ho H, Lee W, Lin C, Kuo C. Interactive effect of exercise training and growth hormone administration on glucose tolerance and muscle GLUT4 protein expression in rats. J Biomed Sci 2003;10:689-696.

Huang S, Czech M. The GLUT4 glucose transporter. Cell Metab. 2007; 5: 237-252.

Ivy J. Muscle insulin resistance amended with exercise training: role of GLUT4 expression. Med Sci Sports Exerc 2004;36:12071211.

Kastorini C, Panagiotakos D. Mediterranean diet and diabetes prevention: Myth or fact? . World J Diabetes. 2010;1:65-67.
Katharine E, Hall K, Matthew W, McDonal, Kenneth N, Grisé, et al., The role of resistance and aerobic exercise training on insulin sensitivity measures in STZ-induced Type 1 diabetic rodents. Metabolism 2013;62:1485-1494

Kiraly M, Bates H, Yue J, Goche-Montes D, Fediuc S, Park E. Attenuation of type 2 diabetes mellitus in the male Zucker diabetic fatty rat: the effects of stress and non-volitional exercise. Metabolism. 2007;56:732-744.

Kraniou G, Cameron-Smith D, Hargreaves M. Effect of shortterm training on GLUT4 mRNA and protein expression in human skeletal muscle. Exp physiol 2004;89:559-563.

McGee S, Howlett K, Starkie R, Cameron-Smith D, Kemp B, Hargreaves M. Exercise Increases Nuclear AMPK $\alpha 2$ in Human Skeletal Muscle. Diabetes 2003;42:926-928.

Musi N, Fujii N, Hirshman M, Ekberg I, Fröberg S, Ljungqvist O. AMP-Activated Protein Kinase (AMPK) Is Activated in Muscle of Subjects With Type 2 Diabetes During Exercise. Diabetes 2001;50: 921 927.

Ogonovszky H, Berkes I, Kumagai S, Kaneko T, Tahara S, Goto S, et al., The effects of moderate-, strenuous- and over-training on oxidative stress markers, DNA repair, and memory, in rat brain Neurochemistry. 2005;46:635-40.

Parimai M, Ranjan C. The role of AMP kinase in diabetes. Indian J Med Res 2007:125:389-398.

Park S, Kim K, Yoon J, Lee S. Effect of exercise on GLUT4 expression of skeletal muscle in streptozotocin-induced diabetic rats. Physiology. 2011;14:113-122.

Paula E, Durante P, Kirsty J, Mustard K, Park S, William W, et al., Effects of endurance training on activity and expression of AMPactivated protein kinase isoforms in rat muscles. American Journal of Physiology - Endocrinology and Metabolism. 2002;283:178-186.

Richter E, Hargreaves M. Exercise, GLUT4, and skeletal muscle glucose uptake. Physiol Rev. 2013;93:993- 1017.

Shabkhiz F, Ravasi A, Hassan Z, Taghikhani M, Razav T. The effect of aerobic continuous and interval training and detraining on some indexes of the cellular immune system in female wistar rats. Sport Sciences. 2008;1:17-26.

Siamilis S, Jakus J, Nyakas C, Costa A, Mihalik B, Falus A, et al., The effect of exercise and oxidant-antioxidant in terventionon the levels of neurotrophins and free radicals in spinal cord of rats. Spinal Cord. 2009;47:453-457.

Srinivasan K, Visvanad B, Asrad CL, Ramarao P. Combination of high-fat diet-fed and low-dose streptozotocin-treated rat: A model for type 2 diabetes and pharmacological screening. Pharmacological Research. 2005;52:313-320.

Teixeira-Lemos E, Nunes S, Teixeira F, Reis F. Regular physical exercise training assists in preventing type 2 diabetes development: focus on its antioxidant and antiinflammatory properties. Cardiovasc Diabetol 2011;28:10- 12.

Tsai Y, Hou C, Liao Y, Chen C, Lin F, Lee W. Exercise training exacerbates tourniquet ischemia-induced decreases in GLUT4 expression and muscle atrophy in rats. Life Sci 2006;78:2953-2959.

Vannucci S, Moehler-Stec K, Li K, Reynolds T, Clark R, Simpson I. GLUT4 glucose transporter expression in rodent brain: effect of diabetes. Brain Res. 1998;22:1-11.

Zarekar M, Saghebjoo M, Foadodini M, Hedayati M. Combined Effect of Aerobic Training and PistaciaAthlantica Extract on GLUT-4 Protein Expression and Muscle Glycogen in Diabetic Rats. Iranian Journal of Endocrinology and Metabolism. 2014;16:245-253

\section{How to cite this article:}

Afzalpour ME, Yousefi MR, Eivari SHA, Ilbeigi S. Changes in blood insulin resistance, GLUT4 \& AMPK after continuous and interval aerobic training in normal and diabetic rats. J App Pharm Sci, 2016; 6 (09): 076-081. 